\title{
Performance of two rapid diagnostic tests for malaria diagnosis at the China-Myanmar border area
}

\author{
Juan Yan ${ }^{1}$, Nana Li ${ }^{2}$, Xu Wei ${ }^{1}$, Peipei $\mathrm{Li}^{3}$, Zhenjun Zhao ${ }^{3}$, Lili Wang ${ }^{2}$, Siying $\mathrm{Li}^{2}$, Xiaomei Li ${ }^{2}$, Ying Wang ${ }^{4}$, \\ Shuying Li $i^{3}$, Zhaoqing Yang ${ }^{2}$, Bin Zheng ${ }^{5}$, Guofa Zhou ${ }^{6}$, Guiyun Yan ${ }^{6}$, Liwang Cui ${ }^{7}$, Yaming Cao ${ }^{1^{*}}$ and Qi Fan ${ }^{3^{*}}$
}

\begin{abstract}
Background: Rapid diagnostic tests (RDTs) have become an essential tool in the contemporary malaria control and management programmes in the world. This study aims to evaluate the performance of two commonly used RDTs for malaria diagnosis in the China-Myanmar border area.

Methods: A total 606 febrile patients in the China-Myanmar border were recruited to this study and were diagnosed for malaria infections by microscopy, two RDTs tests (Pf/Pan device, and PV/Pf device) and nested PCR.

Results: Malaria parasites were found in 143 patients by microscopy, of which 51, 73, and 19 were Plasmodium falciparum, Plasmodium vivax and P. falciparum/P. vivax mixed infections, respectively. Compared to microscopy, the sensitivity of the Pf/Pan device was $88.6 \%$ for $P$. falciparum and $69.9 \%$ for $P$. vivax with the specificity of $90.4 \%$. For a subset of 350 patients, the sensitivity of the Pf/Pan device and Pv/Pf device for detection of P. falciparum was $87.5 \%$ and $91.7 \%$, respectively; and for detection of $P$. vivax was $72.0 \%$ and $73.8 \%$, respectively. The specificity of the Pf/Pan device and Pv/Pf device was $94.3 \%$ and $96.5 \%$, respectively. Nested PCR detected malaria parasites in 174 of 606 samples, of which 67, 79, two and 26 were $P$. falciparum, $P$. vivax, $P$. ovale and $P$. falciparum/P. vivax mixed infections, respectively. Compared to nested PCR, all other methods had sensitivity below $80 \%$, suggesting that a significant number of cases were missed.

Conclusions: Compared to PCR, both microscopy and RDTs had lower sensitivities. RDTs had similar performance to microscopy for $P$. falciparum diagnosis, but performed worse for $P$. vivax diagnosis. Other RDT products should be selected with higher sensitivity (and good specificity) for both $P$. falciparum and $P$. vivax diagnosis.
\end{abstract}

Keywords: Rapid diagnostic tests (RDTs), Malaria diagnosis, Microscopy, PCR, Sensitivity, Specificity

\section{Background}

Malaria is a highly prevalent disease in tropical and subtropical regions, affecting half of the world's population in 108 countries, resulting in almost one million deaths annually [1]. Malaria in human can be caused by one of five malaria parasites (Plasmodium falciparum, Plasmodium vivax, Plasmodium malariae, Plasmodium ovale and Plasmodium knowlesi), which have different geographical distributions. Plasmodium falciparum causes

\footnotetext{
* Correspondence: yamingcao@yahoo.com.cn; fanqi2002@yahoo.com 'Department of Immunology, College of Basic Medical Sciences, China Medical University, Shenyang, Liaoning, China

${ }^{3}$ Dalian Institute of Biotechnology, Dalian, Liaoning, China

Full list of author information is available at the end of the article
}

the most severe form of the disease and tends to predominate in tropical areas. Plasmodium vivax is the predominant species outside Africa. In recent years, it has been increasingly recognized that $P$. vivax is also associated with severe symptoms [2], which changed the traditional view of this malaria as "benign tertian". The great impact of the 130-435 million $P$. vivax infections each year and the significant lag in research and prevention justifies considering $P$. vivax malaria as a neglected tropical disease $[3,4]$. In many parts of the world, such as Southeast Asia, $P$. vivax occurs sympatrically with $P$. falciparum. Since these two parasites require different drug treatments, accurate diagnosis is required to differentiate these two species in areas of co-existence.

\section{Ciomed Central}


Microscopic examination of Giemsa-stained blood smears under a light microscope remains the gold standard method for malaria diagnosis. However, this technique requires a relatively long observation time and well-trained microscopists. Misdiagnosis often happens in samples with low parasitaemia, especially when drugs are taken inappropriately [5,6]. Furthermore, microscopic diagnosis of $P$. vivax is more challenging, because parasite density during $P$. vivax infections is often low. Recently, the use of molecular methods such as PCR, for the diagnosis of malaria has proved to be highly sensitive and specific $[7,8]$, but drawbacks such as a requirement for equipment, higher cost, and a lengthy procedure limit their routine [9-11]. Malaria rapid diagnostic tests (RDTs) have become very popular in various endemic settings [12], especially in areas where microscopic expertise is lacking. They are now an essential tool in malaria management during the malaria elimination/eradication campaign [13]. However, the wide variety of RDTs and their different performance under different endemic settings suggest that careful comparison of RDTs is needed before mass deployment for diagnosis.

RDTs are designed using antibodies against parasite species-specific or genus-specific antigens, such as P. falciparum-specific histidine-rich protein-2 (PfHRP2) and parasite lactate dehydrogenase ( $\mathrm{pLDH})$. However, the performance of RDTs is easily affected by humidity and extreme temperatures. In addition, persistence of antigens that may remain in the circulation of a patient after treatment may give false positive results [14]. In malaria endemic areas of the Greater Mekong Subregion (GMS), the four human malaria species often co-exist, but with $P$. falciparum and $P$. vivax being the predominant species. In this region, cases of human infected by the monkey malaria parasite P. knowlesi were also reported $[15,16]$. Several types of RDTs have been evaluated in these areas, and most of them had poor performance for low levels of parasitaemia (e.g., $<500$ parasites $/ \mu \mathrm{L}$ ), which is especially true for vivax malaria $[17,18]$. In recent years, with extensive supports from the Global Fund to fight AIDS, Tuberculosis and Malaria, the malaria control programme at the ChinaMyanmar border area has been substantially strengthened. Among the control measures is the mass use of RDTs for malaria diagnosis. Specifically, two types of RDTs have been extensively used based on co-existence of P. falciparum and P. vivax in this area: a Pf/Pan test device and $\mathrm{Pv} / \mathrm{Pf}$ test device. However, their performance for malaria diagnosis under this specific endemic setting has not been evaluated. In this study, the performance of the two RDTs was compared with that of microscopy and PCR for the diagnosis of P. falciparum and P. vivax malaria.

\section{Methods}

\section{Study site and patients}

The study was conducted in 2011 in Laiza township area along the China-Myanmar border. Malaria in this region, caused mainly by $P$. falciparum and $P$. vivax infections, is perennial with distinct seasonality, which occurs mostly in the rainy season from April to November. A total of 606 patients attending local malaria clinics and hospital were recruited into the study based on the following criteria: suspected of uncomplicated malaria, having fever with axillary temperature above $37.5^{\circ} \mathrm{C}$ at the time of examination and willing to participate in the study. The study population has a sex ratio of $\sim 1$. Their ages ranged from six months to 88 years with a median of 20.3 years. The relative proportions of patients under 15 years, between 16 and 50 years, and >50 years were $52.9,39.3$, and $7.8 \%$, respectively. The study protocol was reviewed and approved by the Institutional Review Board of Kunming Medical University. All participants or legal guardians gave written informed consent before entering the study. Finger-prick blood was obtained for blood films, RDTs and PCR.

\section{Microscopic examinations}

Thick and thin blood films were prepared from peripheral blood. The slides were stained with Giemsa and screened for the presence of parasites and identification of parasite species. Stained blood films were examined with (a 100×) oil immersion lens. Parasite density was determined by counting the parasites and leucocytes, assuming 8,000 leucocytes/ $\mu \mathrm{L}$ of blood [19]. Blood films were examined by an experienced microscopist who was 'blinded' to the results of additional diagnostic tests. Smears were considered negative if no parasite was seen in 100 oil immersion fields on a thick blood film. All the slides were double checked blindly by a second, independent microscopist and the results were combined. Parasite density was calculated by determining the number of parasites per 200 white blood cells in a thick blood film.

\section{RDTs for malaria}

The RDTs used in this study are One Step Malaria Pf/ Pan test (Wondfo, China) and Malaria Pv/Pf test device (Cat. No. 200317, Tycolpharm Co., Limited, UK). The major target antigens of the Pf/Pan test device are PfHRP2 and pan-pLDH, which are specific for $P$. falciparum and all human Plasmodium species, respectively. The $\mathrm{Pv} / \mathrm{Pf}$ test device is based on PfHRP2 antigen and Pv-pLDH antigen, which are specific for P. falciparum and $P$. vivax, respectively. Five microlitres of fresh whole blood was added to the card pad, and three drops of specific lying agent were added. The RDT result was read in 15-20 min according to the manufacturer's 
Table 1 Primers based on the 18S rRNA gene in malaria parasites for nested PCR diagnosis of malaria infections

\begin{tabular}{|c|c|c|c|}
\hline Species & Primer & Sequence $\left(5^{\prime}-3^{\prime}\right)$ & Size of PCR product (bp) \\
\hline \multirow[t]{2}{*}{ Plasmodium sp. } & rPLU5 & CCTGTTGTTGCCTTAAACTTC & 1100 \\
\hline & rPLU6 & TTAAAATTGTTGCAGTTAAAACG & \\
\hline \multirow[t]{2}{*}{ P. falciparum } & rFAL1 & TTAAACTGGTTGGGAAAACCAAATATATT & 205 \\
\hline & $\mathrm{rFAL} 2$ & ACACAATGAACTCAATCATGACTACCCGTC & \\
\hline \multirow[t]{2}{*}{ P. vivax } & PV18SF & GAATTTCTCTTCGGAGTTTATTC & 419 \\
\hline & PV18SR & GTAGAAAAGGGAAAGGGAAACTGTTA & \\
\hline \multirow[t]{2}{*}{ P. malariae } & PM18SF & GAGACATTCATATATATGAGTGTTCT & 423 \\
\hline & PM18SR & GGGAAAAGAACGTIITTATTAAAAAAAAC & \\
\hline \multirow[t]{2}{*}{ P. ovale } & P018SF & GAAAATTCCTITGGAAATTTCTTAG & 410 \\
\hline & P018SR & GGGAAAAGGACACTATAATGTATC & \\
\hline \multirow[t]{2}{*}{ P. knowlesi } & PK18SF & GAGTTTTCTTTCTCTCCGGAG & 424 \\
\hline & PK18SR & GGGAAAGGAATCACATTTAACGT & \\
\hline
\end{tabular}

instructions and immediately recorded. The test was considered valid when the control line on the immunechromatographic test strip was shown. For the Pf/Pan test device, it was counted as $P$. falciparum-positive if the line detecting P. falciparum-specific PfHRP2 was positive or if the lines detecting both PfHRP2 and pan-pLDH were positive; as non-falciparum if only the pan-pLDH line was positive. For the Pv/Pf test device, it was counted as $P$. falciparum- or $P$. vivax-positive if one of both specific test lines were positive. The readers of RDTs were blinded to the results of microscopy and PCR. All 606 cases in this study were diagnosed by the Malaria Pf/Pan test device. Later, the Malaria Pv/Pf test device was added as a comparison and 350 cases were diagnosed by both RDTs.

\section{Diagnosis by PCR}

Fresh blood samples were spotted on Whatman 3 paper, air-dried at room temperature, and stored individually in a zipper plastic bag at $-20^{\circ} \mathrm{C}$ until use to prevent DNA degradation. Genomic DNA was extracted from dried blood spots using QIAamp DNA Micro Kit (Qiagen) according to the manufacturer's instruction. Nested PCR for Plasmodium species was slightly modified based on previously published work using the small subunit (SSU ) rRNA gene [20,21]. Plasmodium genus-specific primers were shown in Table 1 . For the primary PCR reactions, $2 \mu \mathrm{L}$ of genomic DNA were used in a $25 \mu \mathrm{L}$ reaction with outer primers rPLU5 and rPLU6, and 30 cycles $\left(94^{\circ} \mathrm{C}\right.$ for $1 \mathrm{~min}, 55^{\circ} \mathrm{C}$ for $2 \mathrm{~min}$ and $72^{\circ} \mathrm{C}$ for $2 \mathrm{~min}$ ) were performed. For nested PCR, $2 \mu \mathrm{L}$ of the primary PCR product were used as the template with species-specific primers for the four human malaria species and P. knowlesi in separate reaction tubes. After another 30 cycles of amplification $\left(94^{\circ} \mathrm{C}\right.$ for $40 \mathrm{~s}, 58^{\circ} \mathrm{C}$ for $1 \mathrm{~min}$ and $72^{\circ} \mathrm{C}$ for $2 \mathrm{~min}$ ), the PCR products were separated in $1 \%$ and $2 \%$ agarose gels for primary and nested PCR, respectively. DNA bands were stained with ethidium bromide

Table 2 Interpretation of the results for the Pf/Pan RDT

For $P$. falciparum

Test Line(s) visible

Only Pf or both Pf and Pan line visible

No test line or only Pan line visible

For the non-falciparum species

Test Line(s) visible

only Pan line visible

No test line or Only Pf or both Pf and Pan line visible

\begin{tabular}{|c|c|}
\hline \multicolumn{2}{|c|}{ Species identification by microscopy (or corrected by PCR) } \\
\hline $\begin{array}{l}\text { P. falciparum or as a mixed infection with } P \text {. } \\
\text { falciparum }\end{array}$ & $\begin{array}{l}\text { P. vivax, P. ovale, P. malariae, P. knowlesi /no parasites } \\
\text { detected }\end{array}$ \\
\hline True positive (TP) & Species mismatch**/false positive (FP) \\
\hline False negative/species mismatch* (FN) & True Negative (TN) \\
\hline
\end{tabular}

Species identification by microscopy (or corrected by PCR)

P. vivax, P. ovale, or P. malariae

P. falciparum/no parasites detected

True positive (TP)

Species mismatch*/false positive (FP)

False negative/species mismatch**(FN)

* $P$. falciparum or as a mixed infection with $P$. falciparum diagnosed as non-falciparum species.

** Non-falciparum species diagnosed as $P$. falciparum or as a mixed infection with P. falciparum. 
Table 3 Detection results of malaria infections by the Malaria Pf/Pan test in comparison with microscopy ( $\mathrm{N}=606)$

\begin{tabular}{|c|c|c|c|c|c|c|}
\hline \multirow[t]{2}{*}{ Method } & \multirow[t]{2}{*}{ Species } & \multicolumn{4}{|c|}{ Species identified by microscopy } & \multirow[t]{2}{*}{ Total } \\
\hline & & P. falciparum & P. vivax & P. falciparum $+P$. vivax & Negative & \\
\hline \multirow[t]{5}{*}{ Pf/Pan Test } & P. falciparum & 18 & 3 & 2 & 10 & 33 \\
\hline & Pan (non-falciparum) & 1 & 51 & 1 & 3 & 56 \\
\hline & P. falciparum + Pan & 27 & 13 & 15 & 15 & $70^{*}$ \\
\hline & Negative & 5 & 6 & 1 & 435 & 447 \\
\hline & Total & 51 & 73 & 19 & 463 & 606 \\
\hline
\end{tabular}

* For the Pf/Pan device, these cases had both test lines visible, suggesting they were either $P$. falciparum single infections or $P$. falciparum/non-falciparum mixed infections.

and visualized under a UV light. The primers and expected sizes of the PCR fragments of the SSU rRNA genes are shown in Table 1.

\section{Statistical analysis}

The RDT results were compared with those from microscopy and nested PCR. Sensitivity and specificity were calculated with $95 \%$ confidence intervals (C.I.) in two separate analyses: (1) diagnostic performance of RDTs in comparison with the microscopic method, and (2) comparative diagnostic performances of RDTs in comparison with the microscopy and PCR as the references. Based on the microscopic results, the RDT results were considered true positive (TP), true negative $(\mathrm{TN})$, false positive (FP), and false negative (FN) using the interpretation criteria presented in Table 2. Sensitivity and specificity were calculated as $\mathrm{TP} /(\mathrm{TP}+\mathrm{FN})$ and $\mathrm{TN} /$ $(\mathrm{TN}+\mathrm{FP})$, respectively. Statistical analysis was performed by the Chi-square test using the SPSS version 15.0 and the significance level was set as $<0.05$.

\section{Results}

Blood samples were collected from a total of 606 patients. All samples were evaluated by the Pf/Pan test device, while a subset of 350 were also evaluated by the Pv/Pf test device. Of the 606 samples, malaria parasites were found in 143 by microscopy; 51, 73, and 19 were P. falciparum, $P$. vivax and $P$. falciparum/P. vivax mixed infections, respectively (Table 3 ). No other malaria parasite species were detected by microscopy. The parasite density ranges of $P$. falciparum were $40-105,920$ parasites $/ \mu L$. For $P$. vivax, the majority of cases had parasite density ranging from 80 to 17,800 parasites $/ \mu \mathrm{L}$. One $P$. vivax case had a deviant parasite density of $>200,000$ parasites $/ \mu \mathrm{L}$ based on leucocyte number probably due to a low leucocyte count in this patient. Because P. falciparum single infections and mixed species infections containing $P$. falciparum cannot be differentiated by the Pf/Pan device, the microscopy results were grouped into " $P$. falciparum and mixed infections with $P$. falciparum" and "Non-falciparum". Based on this interpretation, the Pf/Pan device detected 33 single $P$. falciparum infections (only the PfHRP2 line visible), 56 non-falciparum cases (only the pan-pLDH line visible) and $70 P$. falciparum infections/potentially mixed infections (both lines visible) (Table 3). Compared with the results by microscopy, the Pf/Pan device detected 62 and 51 samples as true positives for P. falciparum and non-falciparum, respectively. From this result, the sensitivity of $\mathrm{Pf} / \mathrm{Pan}$ device was $88.6 \%$ for $P$. falciparum and $69.9 \%$ for $P$. vivax with the specificity of $90.4 \%$ (Table 4 ).

For the subset of 350 samples, microscopy detected 37 samples containing $P$. falciparum, 50 samples containing $P$. vivax and 11 were diagnosed as mixed species infections (Table 5). Since the Pv/Pf device is able to differentiate $P$. falciparum and $P$. vivax infections, the microscopy results were grouped into "infections containing P. falciparum" and "infections containing P. vivax". The $\mathrm{Pv} / \mathrm{Pf}$ device detected 40 single $P$. falciparum infections, $42 P$. vivax infections, and 8 mixed species infections (Table 5). Compared to microscopy, the $\mathrm{Pv} / \mathrm{Pf}$ device detected 44 and 45 samples as true positives for $P$. falciparum and $P$. vivax, respectively, whereas the Pf/Pan device detected 42 and 36 samples as true positives for $P$. falciparum and $P$. vivax, respectively. The sensitivity of Pf/Pan device and Pv/Pf device for detection of $P$. falciparum was $87.5 \%$ and $91.7 \%$, respectively; and for detection of $P$. vivax was $72.0 \%$ and $73.8 \%$, respectively (Table 4 ). The specificity of $\mathrm{Pf} / \mathrm{Pan}$ device and $\mathrm{Pv} / \mathrm{Pf}$ device was $94.3 \%$ and $96.5 \%$, respectively (Table 4 ).

It has been observed that some RDTs do not perform well when the parasite density is below 500 parasites $/ \mu \mathrm{L}$.

Table 4 Performance of two RDTs for detection of $\boldsymbol{P}$. falciparum and $P$. vivax with microscopy as the gold standard

\begin{tabular}{llll}
\hline & $\begin{array}{l}\text { Pf/Pan } \\
\text { (N=606) }\end{array}$ & $\begin{array}{l}\text { Pf/Pan } \\
(\mathbf{N}=350)\end{array}$ & $\begin{array}{l}\text { Pv/Pf } \\
\text { (N=350) }\end{array}$ \\
\hline $\begin{array}{l}\text { Sensitivity for } \\
\begin{array}{l}\text { P. falciparum } \\
\text { Sensitivity for P. vivax } \\
\text { (non-falciparum for }\end{array}\end{array}$ & $88.6(85.9-91.3)$ & $87.5(83.8-91.2)$ & $91.7(88.5-94.8)$ \\
Pf/Pan) & & & \\
Specificity & $90.4(87.8-93.1)$ & $94.3(91.4-97.1)$ & $96.5(94.3-98.7)$ \\
\hline
\end{tabular}

Data are presented as percentage ( $95 \%$ confidence interval; $\mathrm{Cl}$ ). 
Table 5 Detection results of malaria infections by Malaria Pv/Pf test and Malaria Pf/Pan test in comparison with the microscopic method $(\mathrm{N}=350)$

\begin{tabular}{|c|c|c|c|c|c|c|c|}
\hline \multirow[t]{2}{*}{ Method } & \multirow[t]{2}{*}{ Species } & \multicolumn{5}{|c|}{ Species identified by microscopy } & \multirow[t]{2}{*}{ Total } \\
\hline & & P. falciparum & P. vivax & P. falciparum $+P$. vivax & P. ovale & Negative & \\
\hline \multirow[t]{4}{*}{ Pv/Pf Test } & P. falciparum & 32 & 1 & 6 & 0 & 1 & 40 \\
\hline & P. vivax & 0 & 39 & 0 & 0 & 3 & 42 \\
\hline & P. falciparum + P. vivax & 2 & 2 & 4 & 0 & 0 & 8 \\
\hline & Negative & 3 & 8 & 1 & 0 & 248 & 260 \\
\hline \multirow[t]{5}{*}{ Pf/Pan Test } & P. falciparum & 17 & 1 & 2 & 0 & 2 & 22 \\
\hline & Pan(non-falciparum) & 0 & 36 & 0 & 0 & 2 & 38 \\
\hline & P. falciparum + Pan & 15 & 8 & 8 & 0 & 2 & $33^{*}$ \\
\hline & Negative & 5 & 5 & 1 & 0 & 246 & 257 \\
\hline & Total & 37 & 50 & 11 & 0 & 252 & 350 \\
\hline
\end{tabular}

* For the Pf/Pan device, these cases had both test lines visible, suggesting they were either $P$. falciparum single infections or $P$. falciparum/non-falciparum mixed infections.

For P. falciparum, both RDTs had significantly higher sensitivity for cases with a parasite density above 500 parasites $/ \mu \mathrm{L}(>92 \%)$ than those with a density below 500 parasites $/ \mu \mathrm{L}(<75.0 \%)$ (Table 6). However, for P. vivax malaria, regardless of the parasite density, both RDTs displayed sensitivity of below 79\%. Both RDTs had similar detection limits; they were $>240$ and $>320$ parasites $/ \mu \mathrm{L}$ for $P$. falciparum and $P$. vivax, respectively.

To further evaluate the performance of these two RDTs, all 606 samples were examined by nested PCR. Nested PCR detected malaria parasites in 174 samples, of which 67, 79, two and 26 were $P$. falciparum, $P$. vivax, $P$. ovale and $P$. falciparum $/ P$. vivax mixed infections, respectively. No P. knowlesi infections were detected by PCR (Table 7). For the subset of 350 samples, 113 samples were identified by nested PCR as infection with malaria parasites. Of which, 40, 52, and 21 were $P$. falciparum, $P$. vivax, and $P$. falciparum/P. vivax mixed infections, respectively (Table 8 ). Among all detection methods, PCR was the most sensitive one. If PCR was used as the gold standard, the sensitivity of microscopy for P. falciparum and P. vivax was $71.0 \%$ and $73.3 \%$ in 606 detected samples, and $75.4 \%$ and $74.0 \%$ in the 350 subset samples, respectively (Table 9 ). The sensitivity of Pf/Pan test for P. falciparum and P. vivax was $81.7 \%$ and $64.6 \%$ in 606 detected samples, and $77.1 \%$ and
$63.5 \%$ in the 350 subset samples, respectively (Table 9). For the subset of 350 samples analyzed by Pv/Pf devices, the sensitivity was $72.1 \%$ for P. falciparum and $58.9 \%$ for $P$. vivax, respectively (Table 9). Microscopy and the two RDTs had similar levels of specificity ranging from $94.7 \%$ to $96.3 \%$ (Table 9). It is noteworthy that although the $\mathrm{Pf} / \mathrm{Pan}$ device is designed to identify all human malaria parasite species, the two $P$. ovale infections identified by PCR were missed by this device and by microscopy, possibly due to the low parasitaemia in the P. ovale infections (containing 120 parasites $/ \mu \mathrm{L}$ and 320 parasites $/ \mu \mathrm{L}$, respectively). Re-examination of the $P$. ovale slides by microscopy did detect $P$. ovale-parasitized erythrocytes (Figure 1).

According to the microscopy results corrected by PCR, four and eight cases were false negative in 606 samples examined by the Pf/Pan test device for $P$. falciparum and $P$. vivax, respectively (Table 3 ). When mixed infections were excluded, three out of four $P$. falciparum cases had lower parasite density $(<480$ parasites $/ \mu \mathrm{L})$. Only one P. falciparum case with higher parasite density $(8,800$ parasites $/ \mu \mathrm{L}$ ) was not detected by the Pf/Pan test device, but was positive by the Pv/Pf test device. Five out of eight P. vivax cases were lower parasite density ( $<560$ parasites/ $\mu \mathrm{L})$. The remaining three $P$. vivax cases with higher parasite density $(1,880 / \mu \mathrm{L}, 2,760 / \mu \mathrm{L}$ and $15,840 / \mu \mathrm{L})$ were

Table 6 Comparative sensitivity of the two RDTs for detection of $P$. falciparum and $P$. vivax in comparison with the microscopic method, categorized according to parasite density

\begin{tabular}{|c|c|c|c|c|c|c|c|}
\hline & \multirow{2}{*}{$\begin{array}{l}\text { Density } \\
\text { (Parasites/ } \mu \mathrm{L} \text { ) }\end{array}$} & \multicolumn{2}{|c|}{$\mathrm{Pf} / \operatorname{Pan}(\mathrm{N}=606)$} & \multicolumn{2}{|c|}{$\mathrm{Pf} / \operatorname{Pan}(\mathrm{N}=350)$} & \multicolumn{2}{|c|}{$\mathrm{Pv} / \mathrm{Pf}(\mathrm{N}=350)$} \\
\hline & & TP (N) & Sensitivity (\%) & TP (N) & Sensitivity (\%) & TP (N) & Sensitivity (\%) \\
\hline \multirow[t]{2}{*}{ P. falciparum } & $>500$ & 51 & $92.7(86.0-99.4)$ & 34 & $94.4(87.3-100)$ & 35 & $97.2(92.1-100)$ \\
\hline & $<500$ & 11 & $73.3(69.4-77.3)$ & 8 & $66.7(61.0-72.4)$ & 9 & $75.0(70.1-80.2)$ \\
\hline \multirow[t]{2}{*}{ P. vivax (or non-falciparum) } & $>500$ & 40 & $69.0(56.7-81.2)$ & 28 & $71.8(57.1-86.5)$ & 36 & $78.3(63.2-93.2)$ \\
\hline & $<500$ & 11 & $73.3(69.4-77.3)$ & 8 & $72.7(67.4-78.1)$ & 9 & $60.0(54.0-66.0)$ \\
\hline
\end{tabular}

Sensitivity is presented as percentage $(95 \%$ confidence interval; $C \mathrm{I}) . T P$ true positive. 
Table 7 Detection results of malaria infections by microscopy and Malaria Pf/Pan test in comparison with the Nested PCR ( $\mathrm{N}=606)$

\begin{tabular}{|c|c|c|c|c|c|c|c|}
\hline \multirow[t]{2}{*}{ Method } & \multirow[t]{2}{*}{ Species } & \multicolumn{5}{|c|}{ Species identified by nested PCR } & \multirow[t]{2}{*}{ Total } \\
\hline & & P. falciparum & $P$. vivax & P. falciparum $+P$. vivax & P. ovale & Negative & \\
\hline \multirow[t]{5}{*}{ Microscopy } & P. falciparum & 39 & 2 & 9 & 0 & 1 & 51 \\
\hline & P. vivax & 1 & 61 & 9 & 0 & 2 & 73 \\
\hline & P. falciparum $+P$. vivax & 12 & 1 & 6 & 0 & 0 & 19 \\
\hline & P. ovale & 0 & 0 & 0 & 0 & 0 & 0 \\
\hline & Negative & 15 & 15 & 2 & 2 & 429 & 463 \\
\hline \multirow[t]{5}{*}{ Pf/Pan Test } & P. falciparum & 16 & 1 & 7 & 1 & 8 & 33 \\
\hline & Pan(non-falciparum) & 0 & 51 & 5 & 0 & 0 & 56 \\
\hline & P. falciparum + Pan & 40 & 10 & 13 & 0 & 7 & $70^{*}$ \\
\hline & Negative & 11 & 17 & 1 & 1 & 417 & 447 \\
\hline & Total & 67 & 79 & 26 & 2 & 432 & 606 \\
\hline
\end{tabular}

* For the Pf/Pan device, these cases had both test lines visible, suggesting they were either $P$. falciparum single infections or $P$. falciparum/non-falciparum mixed infections.

not detected by the Pf/Pan test device. In the subset of 350 samples examined by the $\mathrm{Pv} / \mathrm{Pf}$ test device (mixed infection not included, Table 5), two P. falciparum cases with lower parasite density $(<480$ parasites $/ \mu \mathrm{L})$ were false negative. Nine $P$. vivax cases were the false negative, of which three had lower parasite density $(<320$ parasites $/ \mu \mathrm{L})$, and three cases (parasite density of $1,320 / \mu \mathrm{L}, 2080 / \mu \mathrm{L}$, $7,280 / \mu \mathrm{L}$ ) were positive by the Pf/Pan test device. The other three cases with higher parasite density $(1,880 / \mu \mathrm{L}$, $2,760 / \mu \mathrm{L}$ and $15,840 / \mu \mathrm{L}$ ) were not detected by both RDTs.

False positive and wrong species identification were observed with both RDTs. In all 606 samples (identified by microscopy and corrected by PCR, mixed infection not included) examined by the $\mathrm{Pf} / \mathrm{Pan}$ test device, one $P$. vivax case, one $P$. ovale case, and eight negative cases showed the PfHRP2 line. Ten $P$. vivax cases showed not only the pan-pLDH line, but the PfHRP2 line. Seven negative cases showed both PfHRP2 and pan-pLDH lines (Table 7). In the subset of 350 samples analysed by the Pv/Pf test, two negative cases showed the PfHRP2 line. One P. falciparum case showed the Pv-pLDH line only. Two $P$. vivax cases showed not only the Pv-pLDH line, but the PfHRP2-line. Six $P$. falciparum cases (parasite density ranging from 240 to 34,400 parasites $/ \mu \mathrm{L}$ ) showed not only the PfHRP2 line,

Table 8 Detection results of malaria infections by microscopy, Malaria Pf/Pan test and Malaria Pv/Pf test in comparison with the Nested PCR ( $\mathrm{N}=350)$

\begin{tabular}{|c|c|c|c|c|c|c|c|}
\hline \multirow[t]{2}{*}{ Method } & \multirow[t]{2}{*}{ Species } & \multicolumn{5}{|c|}{ Species identified by Nested PCR } & \multirow[t]{2}{*}{ Total } \\
\hline & & P. falciparum & P. vivax & P. falciparum $+P$. vivax & P. ovale & Negative & \\
\hline \multirow[t]{5}{*}{ Microscopy } & P. falciparum & 29 & 1 & 6 & 0 & 1 & 37 \\
\hline & P. vivax & 0 & 40 & 9 & 0 & 1 & 50 \\
\hline & P. falciparum + P. vivax & 6 & 0 & 5 & 0 & 0 & 11 \\
\hline & P. ovale & 0 & 0 & 0 & 0 & 0 & 0 \\
\hline & Negative & 5 & 11 & 1 & 0 & 235 & 252 \\
\hline \multirow[t]{4}{*}{ Pv/Pf Test } & P. falciparum & 26 & 0 & 12 & 0 & 2 & 40 \\
\hline & P. vivax & 1 & 33 & 8 & 0 & 0 & 42 \\
\hline & $P$. falciparum $+P$. vivax & 6 & 2 & 0 & 0 & 0 & 8 \\
\hline & Negative & 7 & 17 & 1 & 0 & 235 & 260 \\
\hline \multirow[t]{5}{*}{ Pf/Pan Test } & P. falciparum & 14 & 0 & 6 & 0 & 2 & 22 \\
\hline & Pan(non-falciparum) & 0 & 33 & 5 & 0 & 0 & 38 \\
\hline & P. falciparum + Pan & 18 & 5 & 9 & 0 & 1 & $33^{*}$ \\
\hline & Negative & 8 & 14 & 1 & 0 & 234 & 257 \\
\hline & Total & 40 & 52 & 21 & 0 & 237 & 350 \\
\hline
\end{tabular}

* For the Pf/Pan device, these cases had both test lines visible, suggesting they either $P$. falciparum single infections or $P$. falciparum/non-falciparum mixed infections. 
Table 9 Performance of different diagnosis methods with the nested PCR method as the gold standard

\begin{tabular}{llllll}
\hline & Microscopy (N=606) & Pf/Pan (N=606) & Microscopy (N=350) & Pv/Pf (N=350) & Pf/Pan (N=350) \\
\hline Sensitivity for P. falciparum & $71.0(67.0-74.9)$ & $81.7(78.4-85.1)$ & $75.4(70.4-80.4)$ & $72.1(66.9-77.3)$ & $77.1(72.2-81.9)$ \\
Sensitivity for P. vivax & $73.3(69.5-77.2)$ & $64.6(60.5-68.6)$ & $74.0(68.8-79.1)$ & $58.9(53.1-64.7)$ & $63.5(58.0-68.9)$ \\
Specificity & $95.8(93.9-97.6)$ & $92.9(90.5-95.3)$ & $96.3(93.9-98.7)$ & $95.5(92.9-98.1)$ & $94.7(92.0-97.5)$ \\
\hline
\end{tabular}

Data are presented as percentage ( $95 \%$ confidence interval; $\mathrm{Cl})$.

but also the Pv-pLDH line (Table 8). It has been reported that $P$. falciparum infections with high parasite densities may generate a false positive Pv-pLDH line [22-25]. For the $\mathrm{Pv} / \mathrm{Pf}$ test devices, six out of $40 \mathrm{P}$. falciparum (corrected by PCR) with the Pv-pLDH line visible showed that cross reactions between different species occurred although parasite densities of some P. falciparum infections were not high. From these comparisons, the specificities of these devices need further improvement for areas with both $P$. falciparum and $P$. vivax malaria.

Since P. falciparum and $P$. vivax infections are treated with different drugs, it would be important to compare the different methods for detecting mixed species infections. PCR, microscopy and Pv/Pf test device detected 21, 11, and eight mixed-species infections in the 350 subset samples (Table 8), corresponding to $18.6 \%, 11.2 \%$ and $8.9 \%$ of positive cases detected by the respective methods.

\section{Discussion}

RDTs are playing an essential role in the current malaria control/elimination campaign worldwide. In this study, the performance of two RDT devices commonly used at the China-Myanmar border area was evaluated. Compared to diagnosis by microscopy, both the Pf/Pan and $\mathrm{Pv} / \mathrm{Pf}$ devices showed higher sensitivity $(>87 \%)$ for detecting $P$. falciparum infections, whereas the sensitivity for detecting $P$. vivax infections was much lower $(<74 \%)$. Both RDTs had similar levels of detection limits, and their performance was poor for infections with parasite densities below 500 parasites/ $\mu \mathrm{L}$ for $P$. falciparum infections. Besides, since both devices rely on the PfHRP2 antigen for the detection of $P$. falciparum, pfhrp 2 gene deletions will lead to false-negative results [26]. A recent report showing up to $40 \%$ of $P$. falciparum parasites with pfhrp 2 deletions in South America [27] indicate that such RDTs would not perform well in these regions. It certainly warrants more detailed investigations in other malaria endemic regions.

When PCR was used as the gold standard, the detection sensitivity of both RDTs was reduced especially for the detection of $P$. vivax infections $(<64 \%)$, although the specificity of the all detection methods remained above $92 \%$. In addition, the sensitivity of conventional microscopy was also low, ranging from $71.0 \%$ to $77.4 \%$ for detecting both parasite species. This result is worrisome; since nearly $30 \%$ of the malaria cases was not correctly diagnosed by microscopy or RDTs and subsequently treated, these patients may constitute an important reservoir for transmission. Whereas low parasite density might be the most important limiting factor for microscopic diagnosis [6,28], the varying skills and experience of microscopists may also be responsible for the lower sensitivity of diagnosis [29]. In the past, the Pf/Pan test (Wondfo) has been evaluated for diagnosis of $P$. falciparum only and showed highly satisfactory result [30]. Recently, a CareStart $^{\mathrm{TM}}$ kit (Pf/Pan) was evaluated in the nearby Yunnan province of China, which showed comparable results to microscopy for diagnosing $P$. falciparum and P. vivax infections [31]. Therefore, further evaluations of RDTs are needed to select better diagnostic tests with improved accuracy in this region.

Overall, both RDTs have good sensitivity for detecting P. falciparum infections, but the sensitivity for detecting
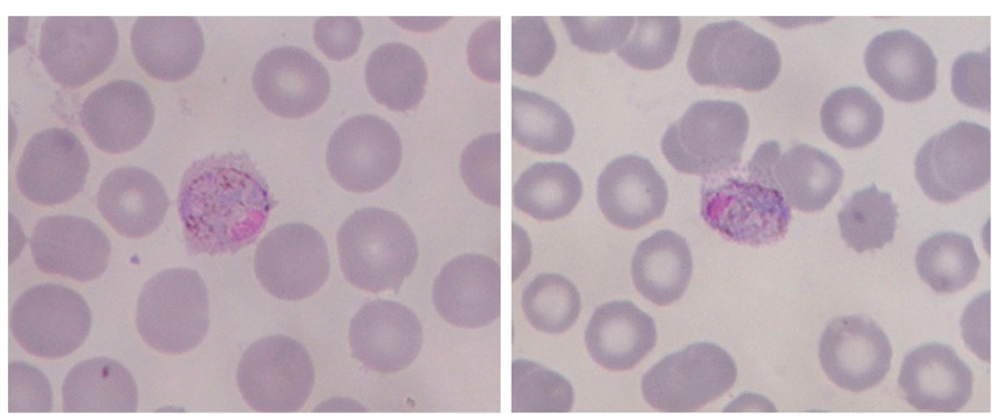

Figure 1 Blood smears showing parasitized erythrocytes by $\boldsymbol{P}$. ovale in two malaria patients. The two patients with febrile illness attended a malaria clinic at the China-Myanmar border and were diagnosed for malaria. Both cases were only detected by PCR but missed by microscopy and RDTs. 
$P$. vivax was much lower. This could be due to lower parasite density for $P$. vivax since this parasite selectively invades reticulocytes. However, comparison of the two RDTs showed that the sensitivities for $P$. vivax detection were not different between the high and low parasite densities (Table 6). This observation is difficult to explain and could be due to the lower number of infections analyzed in the $<500$ parasites $/ \mu \mathrm{L}$ group. Furthermore, the arbitrary selection of 500 parasites $/ \mu \mathrm{L}$ as the border line between high and low parasite densities may not be appropriate for $P$. vivax. In addition, the sensitivity to $P$. vivax may be affected by other factors. Therefore, better RDTs with significantly improved sensitivity for $P$. vivax are needed.

One important criterion for the selection of RDTs in the GMS is the ability to detect other human malaria species in addition to $P$. falciparum. The advantage of the $\mathrm{Pf} / \mathrm{Pan}$ test is that it is designed to detect four human parasite species, which co-exist in the GMS. However, this device failed to detect the two $P$. ovale infections, suggesting that further improvement in sensitivity for detecting other malaria species is needed. It is also worth to note that the PCR method failed to identify any $P$. knowlesi infections, which is in stark contrast to the eaerlier report of some $30 \%$ of malaria cases in this region as mixed infections with P. knowlesi [16]. Therefore, the significantly lower prevalence of other malaria parasite species in this region and the generally low sensitivity of the Pf/Pan test for diagnosis of $P$. ovale and $P$. malariae suggest that such an advantage is hardly of any importance in this region. In comparison, the $\mathrm{Pv} / \mathrm{Pf}$ test is specific for P. falciparum and $P$. vivax, which make up the majority of malaria infections in the GMS, although it would certainly miss detecting $P$. malariae and $P$. ovale infections. Because $P$. falciparum and $P$. vivax require different antimalarial treatments, correct diagnosis of mixed infections should be an important criterion for the selection of an RDT. Even though the Pv/Pf device is able to detect $P$. falciparum and $P$. vivax mixed infection, the detection rate was low and the eight mix infections detected by this device were all misdiagnosed when compared to the PCR result. This result may be due to reduced density of one parasite species in the presence of another due to competition in the same host. Consequently, with the current anti-malarial drug policy of this region, misdiagnosis of mixed species infections as single species infections would lead to improper drug treatments.

\section{Conclusions}

The aim of this study was to evaluate the performance of two RDTs for malaria diagnosis at the China-Myanmar border area. PCR method was much more sensitive than microscopy and RDTs. Compared to microscopy, both
RDTs demonstrated similar sensitivities for detecting $P$. falciparum infections but reduced sensitivity for detecting $P$. vivax infections. Neither of them had satisfactory results in detecting mixed species infections. Therefore, methods with improved sensitivity for diagnosing $P$. vivax malaria are needed. False negative results for the diagnosis of $P$. falciparum malaria call for further investigations on potential deletion of $p f h r p 2$ gene in this malaria-endemic region.

\section{Competing interests}

The authors declare that they have no competing interests.

\section{Authors' contributions}

YJ, LN, WX, LP and ZZ carried out the experimental work and data analysis. $W L, L S$ and $Z G$ participated in data analysis. YJ performed manuscript writing. YC, QF and LC conceived the study and participated in the design of the study. All authors read and approved the final manuscript.

\section{Acknowledgements}

This work was supported by grant from the National Institute of Allergy and Infectious Diseases, National Institutes of Health (U19 Al089672).

\section{Author details}

${ }^{1}$ Department of Immunology, College of Basic Medical Sciences, China Medical University, Shenyang, Liaoning, China. ${ }^{2}$ Department of Parasitology, Kunming Medical University, Kunming, Yunnan, China. ${ }^{3}$ Dalian Institute of Biotechnology, Dalian, Liaoning, China. ${ }^{4}$ Third Military Medical University, Chongqing, China. ${ }^{5}$ Institute of Parasitic Diseases, Shanghai, China.

${ }^{6}$ University of California, Irvine, CA, USA. ${ }^{7}$ Department of Entomology, Pennsylvania State University, University Park, PA, USA.

Received: 19 September 2012 Accepted: 18 February 2013 Published: 22 February 2013

\section{References}

1. World Health Organization: World malaria report 2011. Geneva: World Health Organization; 2011.

2. Rogerson SJ, Carter R: Severe vivax malaria: newly recognised or rediscovered. PLoS Med 2008, 5:e136.

3. Price RN, Tjitra E, Guerra CA, Yeung S, White NJ, Anstey NM: Vivax malaria: neglected and not benign. Am J Trop Med Hyg 2007, 77:79-87.

4. Baird JK: Neglect of Plasmodium vivax malaria. Trends Parasitol 2007, 23:533-539.

5. Amexo M, Tolhurst R, Barnish G, Bates I: Malaria misdiagnosis: effects on the poor and vulnerable. Lancet 2004, 364:1896-1898.

6. Snounou G, Viriyakosol S, Jarra W, Thaithong S, Brown KN: Identification of the four human malaria parasite species in field samples by the polymerase chain reaction and detection of a high prevalence of mixed infections. Mol Biochem Parasitol 1993, 58:283-292.

7. Snounou G, Viriyakosol S, Zhu XP, Jarra W, Pinheiro L, do Rosario VE, Thaithong S, Brown KN: High sensitivity of detection of human malaria parasites by the use of nested polymerase chain reaction. Mol Biochem Parasitol 1993, 61:315-320.

8. Kho WG, Chung JY, Sim EJ, Kim MY, Kim DW, Jongwutiwes S, Tanabe K: A multiplex polymerase chain reaction for a differential diagnosis of Plasmodium falciparum and Plasmodium vivax. Parasitol Int 2003, 52:229-236.

9. Makler MT, Palmer CJ, Ager AL: A review of practical techniques for the diagnosis of malaria. Ann Trop Med Parasitol 1998, 92:419-433.

10. Farnert A, Arez AP, Correia AT, Bjorkman A, Snounou G, do Rosario V: Sampling and storage of blood and the detection of malaria parasites by polymerase chain reaction. Trans $R$ Soc Trop Med Hyg 1999, 93:50-53.

11. Hwang SY, Kim SH, Lee GY, Hang VT, Moon CS, Shin JH, Koo WL, Kim SY, Park HJ, Park HO, Kho WG: A novel real-time PCR assay for the detection of Plasmodium falciparum and Plasmodium vivax malaria in low parasitized individuals. Acta Trop 2011, 120:40-45.

12. Lubell $Y$, Reyburn $H$, Mbakilwa $H$, Mwangi R, Chonya K, Whitty CJ, Mills A: The cost-effectiveness of parasitologic diagnosis for malaria-suspected 
patients in an era of combination therapy. Am J Trop Med Hyg 2007, 77:128-132

13. Bisoffi Z, Gobbi F, Angheben A, Van den Ende J: The role of rapid diagnostic tests in managing malaria. PLoS Med 2009, 6:1000063.

14. Wongsrichanalai C, Barcus MJ, Muth S, Sutamihardja A, Wernsdorfer WH: A review of malaria diagnostic tools: microscopy and rapid diagnostic test (RDT). Am J Trop Med Hyg 2007, 77:119-127.

15. Putaporntip C, Hongsrimuang T, Seethamchai S, Kobasa T, Limkittikul K, Cu $L$, Jongwutiwes S: Differential prevalence of Plasmodium infections and cryptic Plasmodium knowlesi malaria in humans in Thailand. $J$ Infect Dis 2009, 199:1143-1150.

16. Jiang N, Chang Q, Sun X, Lu H, Yin J, Zhang Z, Wahlgren M, Chen Q: Coinfections with Plasmodium knowlesi and other malaria parasites, Myanmar. Emerg Infect Dis 2010, 16:1476-1478.

17. Coleman RE, Maneechai N, Ponlawat A, Kumpitak C, Rachapaew N, Miller RS, Sattabongkot J: Failure of the OptiMAL rapid malaria test as a tool for the detection of asymptomatic malaria in an area of Thailand endemic for Plasmodium falciparum and P. vivax. Am J Trop Med Hyg 2002, 67:563-565.

18. Coleman RE, Maneechai N, Rachapaew N, Kumpitak C, Soyseng V, Miller RS, Thimasarn K, Sattabongkot J: Field evaluation of the ICT Malaria Pf/Pv immunochromatographic test for the detection of asymptomatic malaria in a Plasmodium falciparum/vivax endemic area in Thailand. Am J Trop Med Hyg 2002, 66:379-383.

19. World Health Organization: Basic Malaria Microscopy - Part I .Learner's Guide. 2nd edition. 2010. http://whqlibdoc.who.int/publications/2010/ 9789241547826_eng.pdf.

20. Buppan P, Putaporntip C, Pattanawong U, Seethamchai S, Jongwutiwes S: Comparative detection of Plasmodium vivax and Plasmodium falciparum DNA in saliva and urine samples from symptomatic malaria patients in a low endemic area. Malar J 2010, 9:72.

21. Johnston SP, Pieniazek NJ, Xayavong MV, Slemenda SB, Wilkins PP, da Silva $\mathrm{AJ}$ : PCR as a confirmatory technique for laboratory diagnosis of malaria. J Clin Microbiol 2006, 44:1087-1089.

22. Gillet $\mathrm{P}$, Bosselaers K, Cnops L, Bottieau E, Van Esbroeck M, Jacobs J: Evaluation of the SD FK70 malaria Ag Plasmodium vivax rapiddiagnostic test in a non-endemic setting. Malar J 2009, 8:129.

23. Gillet P, van Dijk DP, Bottieau E, Cnops L, Van Esbroeck M, Jacobs J: Test characteristics of the SD FK80 Plasmodium falciparum/Plasmodium vivax malaria rapid diagnostic test in a non-endemic setting. Malar J 2009, 8:262.

24. van Dijk DP, Gillet P, Vlieghe E, Cnops L, van Esbroeck M, Jacobs J: Evaluation of the Palutop +4 malaria rapid diagnostic test in a nonendemic setting. Malar J 2009, 8:293.

25. Maltha J, Gillet P, Cnops L, van den Ende J, van Esbroeck M, Jacobs J: Malaria rapid diagnostic tests: Plasmodium falciparum infections with high parasite densities may generate false positive Plasmodium vivax pLDH lines. Malar J 2010, 9:198.

26. Maltha J, Gamboa D, Bendezu J, Sanchez L, Cnops L, Gillet P, Jacobs J: Rapid diagnostic tests for malaria diagnosis in the Peruvian Amazon: impact of pfhrp2 gene deletions and cross-reactions. PLOS ONE 2012, 7:e43094.

27. Gamboa D, Ho M-F, Bendezu J, Torres K, Chiodini PL, Barnwell JW, Incardona S, Perkins M, Bell D, McCarthy J, Cheng Q: A large proportion of P. falciparum isolates in the Amazon Region of Peru lack pfhrp2 and pfhrp3: implications for malaria rapid diagnostic tests. PLOS ONE 2010, 5:e8091.

28. McManus DP, Bowles J: Molecular genetic approaches to parasite identification: their value in diagnostic parasitology and systematics. Int J Parasitol 1996, 26:687-704.

29. Kahama-Maro J, D'Acremont V, Mtasiwa D, Genton B, Lengeler C: Low quality of routine microscopy for malaria at different levels of the health system in Dar es Salaam. Malar J 2011, 10:332.

30. Peng Y, Wu J, Wang J, Li W, Yu S: Study and evaluation of Wondfo rapid diagnostic kit based on nano-gold immunochromatography assay for diagnosis of Plasmodium falciparum. Parasitol Res 2012, 110:1421-1425.

31. Xiaodong S, Tambo E, Chun W, Zhibin C, Yan D, Jian W, Jiazhi W, Xiaonong Z: Diagnostic performance of CareStart ${ }^{\mathrm{TM}}$ malaria HRP2/pLDH (Pf/pan) combo test versus standard microscopy on falciparum and vivax malaria between China-Myanmar endemic borders. Malar J 2013, 12:6.

doi:10.1186/1475-2875-12-73

Cite this article as: Yan et al:: Performance of two rapid diagnostic tests for malaria diagnosis at the China-Myanmar border area. Malaria Journal 2013 12:73.

\section{Submit your next manuscript to BioMed Central and take full advantage of:}

- Convenient online submission

- Thorough peer review

- No space constraints or color figure charges

- Immediate publication on acceptance

- Inclusion in PubMed, CAS, Scopus and Google Scholar

- Research which is freely available for redistribution 